INGENIERÍA DE MATERIALES

\title{
Estudio de morteros adicionados con catalizador usado de craqueo catalítico (FCC) bajo el efecto de altas temperaturas
}

MATERIALS ENGINEERING

\section{Study of mortars added with fluid catalytic cracking catalyst residue (FCC) under the influence of high temperatures}

\author{
Silvia R. Izquierdo*§, Ruby Mejía de Gutiérrez*, Janneth Torres Agredo** \\ *Escuela de Ingeniería de Materiales, GMC (CENM), Universidad del Valle, Colombia \\ **Facultad de Ingeniería y Administración, Universidad Nacional de Colombia, Palmira \\ §silvia.izquierdo@correounivalle.edu.co,ruby.mejia@correounivalle.edu.co,jtorresa@unal.edu.co
}

(Recibido: 16 de Diciembre de 2013 -Aceptado: 09 de Agosto de 2014)

\begin{abstract}
RESUMEN
Se analizó el efecto de la incorporación de un residuo de la industria petroquímica, denominado catalizador gastado del craqueo catalítico (FCC), en pastas y morteros expuestos a temperaturas de $25^{\circ} \mathrm{C}, 200^{\circ} \mathrm{C}, 300^{\circ} \mathrm{C}, 500^{\circ} \mathrm{C}, 750^{\circ} \mathrm{C}, 1000^{\circ} \mathrm{C}$ y $1200^{\circ} \mathrm{C}$, utilizando como materiales de referencia, además del cemento Portland (OPC), metacaolín (MK) y humo de sílice (HS). Las adiciones fueron incorporadas en un $10 \%$ con respecto a la cantidad de cemento. Se evaluó el efecto de la temperatura de exposición en el peso y resistencia a la compresión, igualmente las modificaciones en el aspecto físico posterior a la exposición térmica. Se realizaron análisis termogravimétricos (TG) y de microscopía electrónica de barrido (SEM), para evidenciar los cambios. Los resultados indican que el FCC actúa positivamente. Se resalta que a temperatura de $750^{\circ} \mathrm{C}$ la resistencia residual de los morteros de $\mathrm{OPC}$ es un $16,53 \%$ de la resistencia a temperatura ambiente, comparado a los morteros adicionados, los cuales alcanzan hasta un $29,24 \%$. El FCC retrasa la aparición de grietas en los elementos expuestos a altas temperaturas comparados a aquellos sin adición; su resistencia residual a $750^{\circ} \mathrm{C}$ es un $26 \%$ superior al material de referencia $(\mathrm{OPC})$.
\end{abstract}

Palabras clave: Altas temperaturas, catalizador de craqueo catalítico, microestructura, morteros de cemento adicionado, resistencia mecánica residual.

\begin{abstract}
In this article, the effect of the addition of a petrochemical industry residue, called spent catalyst from the catalytic cracking (FCC), in cementitious materials exposed to temperatures of $25{ }^{\circ} \mathrm{C}, 200{ }^{\circ} \mathrm{C}, 300{ }^{\circ} \mathrm{C}, 500{ }^{\circ} \mathrm{C}, 750{ }^{\circ} \mathrm{C}, 1000$ ${ }^{\circ} \mathrm{C}$ and $1200{ }^{\circ} \mathrm{C}$ was analyzed. Metakaolin (MK) and silica fume (HS) were used as reference materials. The additions were incorporated into a $10 \%$ replacement of the proportion of cement (OPC) in the mixture. The effects of temperature exposure in the weight change and compressive strength were evaluated. Also, changes in the physical appearance of the specimens after thermal exposure were studied. Techniques of Thermogravimetric Analysis (TG) and scanning electron microscopy (SEM) were used to analyze the changes. The results indicate that the addition of the FCC acts positively on the performance of Portland cement. It is noteworthy that the residual strength of OPC mortars at temperature of $750{ }^{\circ} \mathrm{C}$ is $16.53 \%$ of the strength at room temperature compared to the mortars added, which reach up to $29.24 \%$. The FCC addition delayed the appearance of cracks in the elements exposed to high temperatures compared to those without addition; its residual strength at $750^{\circ} \mathrm{C}$ is $26 \%$ higher than the reference material (OPC).
\end{abstract}

Keywords: Blended cement mortars, catalytic cracking catalyst, high temperature, microstructure, residual mechanical strength. 


\section{Introducción}

El fuego representa uno de los inconvenientes más graves al cual una estructura puede estar sometida a lo largo de su vida en servicio, por lo cual la provisión de medidas de seguridad contra incendios es un aspecto importante a considerar tanto en los materiales utilizados como en el diseño estructural, con el fin de prevenir la pérdida de vidas humanas $\mathrm{y}$ de los bienes. El concreto aunque presenta baja conductividad térmica y alto calor específico, esto no significa que la exposición a altas temperaturas no afecten sus propiedades, ya que además de cambios de color, pueden presentarse modificaciones en su resistencia a la compresión, módulo de elasticidad, densidad y porosidad, entre otros parámetros (Arioz, 2007; Netinger et al., 2011; Uysal et al., 2012; Yuksel et al., 2011; de Souza \& Moreno, 2010; Kodur, 2014)). En el caso de un elemento de concreto reforzado, a pesar de que los coeficientes de dilatación del acero y el concreto son similares a temperatura ambiente $\left(1.2 \times 10^{-5} \mathrm{~m} / \mathrm{m}^{\circ} \mathrm{C}\right.$ para el acero y $1.0 \times 10^{-5} \mathrm{~m} /$ $\mathrm{m}^{\circ} \mathrm{C}$ para el concreto), a medida que se incrementa la temperatura, los coeficientes se separan hasta el punto que el del acero llega a ser 30 veces superior al del concreto y por ello se producen tensiones elevadas que generan el desprendimiento del recubrimiento quedando el acero expuesto, lo cual contribuye a una mayor probabilidad de colapso de la estructura (Fernández Canovas, 1994).

Los factores que influencian el desempeño de morteros y concretos al ser expuestos a altas temperaturas están directamente relacionados a los materiales y efectos ambientales, entre estos: el proceso térmico (tiempo, temperatura, velocidad de calentamiento y/o enfriamiento); la naturaleza y porosidad de los agregados; el estado de humedad inicial del concreto; el nivel de esfuerzos al cual está sometido el concreto; y otros propios del elemento (espesor, tipo de refuerzo, dimensiones) (Sarshar \& Khoury, 1993; Ahmed et al., 1992; Poon et al., 2001; Poon \& Azhar, 2003; Netinger et al., 2011; Balazs \& Lubloy, 2012).

En lo que respecta a los materiales utilizados en el concreto, los agregados comúnmente usados son térmicamente estables hasta $350^{\circ} \mathrm{C}$ pero en función de su composición a mayores temperaturas su desempeño puede variar. Por ejemplo, en agregados silíceos pueden presentarse transformaciones de $\alpha$-cuarzo (trigonal) a $\beta$-cuarzo (hexagonal) a 573 ${ }^{\circ} \mathrm{C}$ con un aumento de volumen $(\sim 5,7 \%)$ (Balazs \& Lubloy, 2012); a temperaturas más altas, a partir de $600{ }^{\circ} \mathrm{C}$, agregados calcáreos tales como calcita $\left(\mathrm{CaCO}_{3}\right)$, magnesita $\mathrm{MgCO}_{3}$ y/o dolomita $\left(\mathrm{MgCO}_{3} / \mathrm{CaCO}_{3}\right)$ se disocian en óxidos de calcio o magnesio y $\mathrm{CO}_{2}$ e igualmente por encima de $1200{ }^{\circ} \mathrm{C}$ algunos agregados, por ejemplo basalto, muestran desgasificación y expansión (Naus, 2005; Turker et al., 2001). Respecto al cemento Portland, las reacciones de degradación están asociadas con la pérdida de agua por evaporación del agua libre y la deshidratación de los componentes de la pasta de cemento endurecida (Peng et al., 2001; Nijland, 2001; Georgali \& Taskiridis, 2005). Así, cuando el concreto se expone a una temperatura de $100^{\circ} \mathrm{C}$ el agua capilar comienza a evaporarse, entre $200^{\circ} \mathrm{C}$ y $300^{\circ} \mathrm{C}$ la pérdida de agua capilar es completa y cuando se alcanza la temperatura de $300^{\circ} \mathrm{C}$ el agua ubicada entre las capas y la combinada químicamente en el silicato cálcico hidratado (CSH) y los sulfoaluminatos hidratados inicia la evaporación, lo cual se refleja en la disminución en la resistencia mecánica del material y la aparición de las primeras fisuras. A temperaturas alrededor de $400-500{ }^{\circ} \mathrm{C}$ se produce la descomposición de la Portlandita $(\mathrm{CH})$ lo que trae como consecuencia tensiones internas. La descomposición progresiva del CSH con el incremento de la temperatura se asocia a la pérdida total de las resistencias mecánicas (Arioz, 2007).

En general, estos cambios de tipo físico y químico en el concreto expuesto al fuego se consideran en su mayoría de carácter no-reversible y traen como consecuencia el deterioro de los componentes del concreto simple y reforzado generando finalmente la falla estructural. Debido a esto, se han realizado estudios para incrementar la resistencia al fuego del concreto y se han planteado diferentes recomendaciones, entre estas se sugiere la sustitución parcial del cemento con diversos subproductos industriales y algunos minerales, tales como ceniza volante, escoria, humo de sílice y puzolanas volcánicas; en este caso el mejor desempeño al fuego de concretos adicionados 
con puzolanas se atribuye a la disminución del hidróxido de calcio en la mezcla a consecuencia de la reacción puzolánica (Behnood, 2008; Tanyildizi \& Coskun, 2008; Heikal, 2000, 2013; Hossain, 2006; Uysal et al., 2012; Demirboga et al., 2007; Aydin, 2008; Xu et al., 2001), aunque existen resultados contradictorios atribuidos a que la mayor densificación del material genera a altas temperaturas mayor probabilidad al agrietamiento y delaminación (Sarshar \& Khoury, 1993). Como medida de control a este último efecto, otros estudios se han orientado al uso de fibras tales como las de polipropileno (PP), las cuales contribuyen a aumentar la resistencia residual del elemento. Esta acción se atribuye a la fusión de las fibras a temperaturas del orden de $170^{\circ} \mathrm{C}$, lo cual genera canales de expansión que reducen la presión interna del concreto evitando así la delaminación y mayores pérdidas de la resistencia mecánica (Xiao, 2006; Kalifa et al., 2001; Aydin et al., 2008; Behnood \& Ghandehairi, 2009; Pliya et al., 2011).

El presente artículo analiza el desempeño de morteros adicionados con $10 \%$ de un residuo industrial denominado catalizador usado del craqueo catalítico (FCC, por las siglas en inglés de Fluid Catalityc Cracking), al ser expuestos a un rango de temperaturas entre $25^{\circ} \mathrm{C}-1200^{\circ} \mathrm{C}$ al respecto de lo cual no se encuentra literatura publicada; los resultados obtenidos se comparan con los correspondientes de cementos adicionados con metacaolín (MK) y humo de sílice (HS), incorporados en el mismo porcentaje. Este material, procedente del proceso de refinamiento de crudo para la producción de gasolina y otros combustibles, ha venido siendo estudiado como adición puzolánica al cemento con resultados positivos en sus propiedades tanto mecánicas como de durabilidad (Chen et al., 2004; Payá et al., 2003, 2009; Tseng et al., 2005; Torres et al., 2009; Pinto et al., 2007; Su et al., 2000).

\section{Materiales y procedimiento experimental}

\subsection{Caracterización de materiales}

Para la realización del presente estudio, se utilizó un cemento Portland (OPC) y tres tipos de materiales como adición: un residuo del catalizador de craqueo catalítico (FCC), metacaolin (MK) y humo de sílice (HS), estos dos últimos como materiales de referencia. Cabe anotar, que el cemento utilizado (OPC) en las mezclas es Portland Tipo I, pero contiene adición de material calizo. Las características fisicoquímicas de los materiales de adición utilizados y el cemento se presentan en la Tabla 1. La composición química se determinó por fluorescencia de rayos $\mathrm{X}$ en un espectrómetro MagixPro PW-2440 Philips equipado con un tubo de Rodio y potencia máxima de $4 \mathrm{KW}$, y el tamaño de partícula utilizando un granulometro laser Mastersizer 2000 (Malvern Instruments). En esta tabla se observa que el FCC está compuesto en gran parte por alúmina y sílice, en un orden cercano al $90 \%$ y con un tamaño promedio de partícula de $16,15 \mu \mathrm{m}$. La figura 1(a) presenta el difractograma de Rayos X (DRX), correspondiente a cada una de las adiciones utilizadas; en este se aprecia que

Tabla 1. Características químicas y físicas de los materiales utilizados.

\begin{tabular}{ccccc}
\hline Características & FCC & MK & HS & OPC \\
\hline $\mathrm{SiO}_{2}, \%$ & 44,13 & 53,00 & $>90,00$ & 20,20 \\
$\mathrm{Al}_{2} \mathrm{O}_{3}, \%$ & 46,06 & 43,80 & - & 7,00 \\
$\mathrm{Fe}_{2} \mathrm{O}_{3}, \%$ & 0,81 & 0,43 & - & 4,80 \\
$\mathrm{CaO}, \%$ & 0,52 & 0,02 & - & 58,40 \\
$\mathrm{MgO}, \%$ & - & 0,03 & - & - \\
$\mathrm{K}_{2} \mathrm{O}, \%$ & 0,11 & 0,19 & - & - \\
$\mathrm{TiO}_{2}, \%$ & 0,67 & 1,70 & - & - \\
Pérdidas por ignición, \% & 6,50 & 0,46 & 6,00 & 9,60 \\
Densidad $\left(\mathrm{kg} / \mathrm{m}^{3}\right)$ & 2.630 & 2.600 & 2.010 & 3.500 \\
Tamaño medio de partícula $(\mu \mathrm{m})$ & 16,15 & 1,20 & $0,20-0,50$ & 20,67 \\
\hline
\end{tabular}



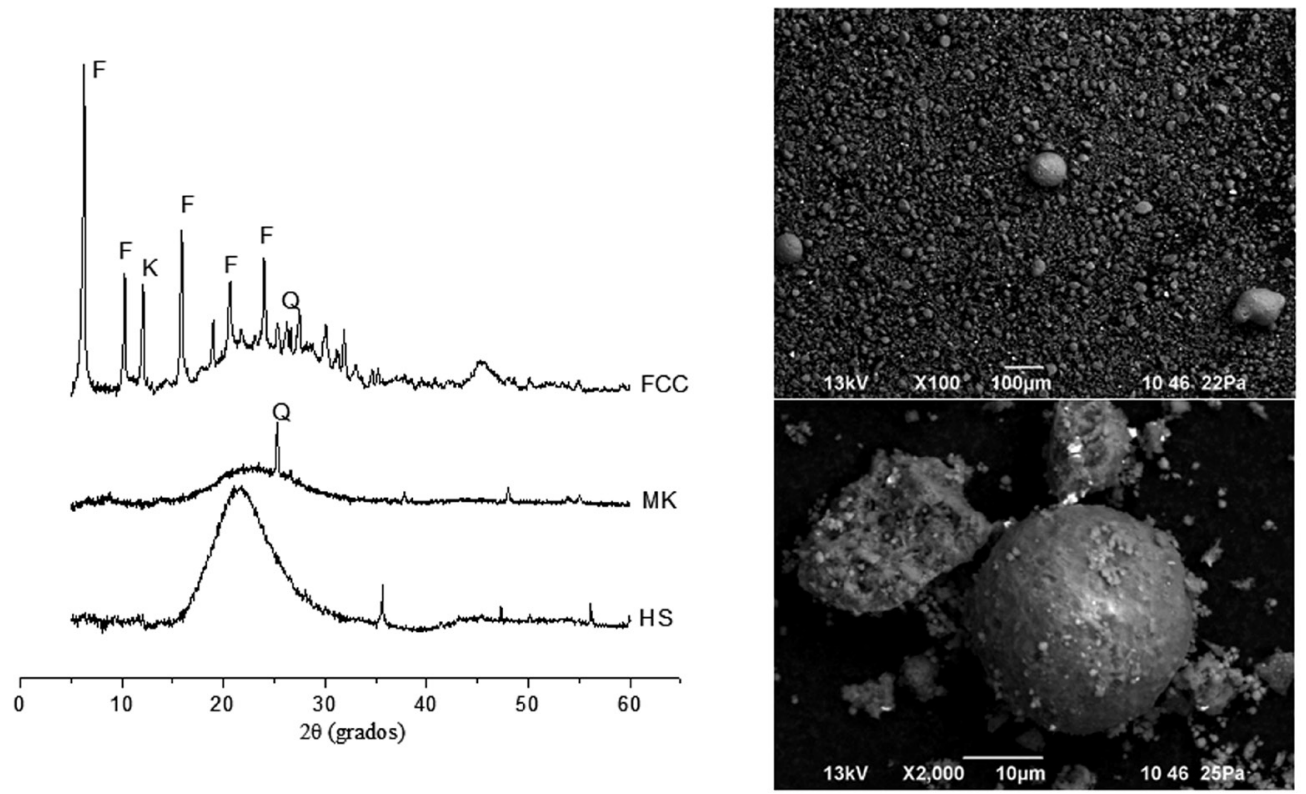

Figura 1. (a) Difractogramas de Rayos X. FCC, MK y HS (F: faujasita, Q: cuarzo, K: caolinita); (b) Micrografia SEM del catalizador gastado del craqueo catalítico (FCC)

el FCC es parcialmente amorfo y cuenta con la presencia de fases cristalinas tales como caolinita $(\mathrm{K})$, cuarzo $(\mathrm{Q})$ y una fase tipo aluminosilicato sódico hidratado de carácter zeolítico similar a la Faujasita (F) de fórmula $\mathrm{Na}_{2}\left[\mathrm{Al}_{2} \mathrm{Si}_{10} \mathrm{O}_{24}\right] \cdot \mathrm{nH}_{2} \mathrm{O}$, esta última relacionada a los picos ubicados en $2 \Theta=6,19^{\circ}, 15,6^{\circ}, 23,58^{\circ}$ (Nan et al., 2000; YunSheng et al., 2005). En el caso del metacaolín y del humo de sílice el difractograma indica un alto grado de amorficidad, dada la presencia de una banda amplia ubicada en el rango $2 \Theta=15-30^{\circ}$.

Adicionalmente se realizó el estudio de la morfología del FCC por medio de microscopia electrónica de barrido (SEM). En la Figura 1(b) se aprecia que las partículas son esféricas o esferoidales, con una alta porosidad, esto le confiere elevada superficie específica y se refleja en el índice de puzolanicidad de la mezcla (OPC $+20 \%$ de FCC) el cual, evaluado con base en la norma ASTM C311, fue de $117 \%$, valor que supera ampliamente lo especificado en la norma ASTM C618 para materiales puzolánicos (75\%).

\subsection{Preparación de muestras para experimentación}

Para el desarrollo de este estudio se elaboraron pastas y morteros con cemento Portland (OPC) y con sustitución de este por catalizador gastado del craqueo catalítico (FCC), metacaolín (MK) y humo de sílice (HS) en proporción del 10\%, para un total de cuatro (4) mezclas. Las pastas se prepararon con una relación agua/cementante (a/C) de 0.35 , donde $\mathrm{C}$ representa la proporción de OPC+Adición, mientras que los morteros se prepararon con relación a/C de 0.5 y una relación cemento: agregado de 1:2,75. El agregado utilizado en este estudio fue arena de Ottawa.

Las muestras fueron curadas por un periodo de 28 días en agua saturada con $\mathrm{Ca}(\mathrm{OH})_{2}$ y 15 días al aire para posteriormente ser expuestas durante dos horas a las temperaturas de $25^{\circ} \mathrm{C}$, $100^{\circ} \mathrm{C}, 200^{\circ} \mathrm{C}, 300^{\circ} \mathrm{C}, 500^{\circ} \mathrm{C}, 750^{\circ} \mathrm{C}, 1000^{\circ} \mathrm{C} \mathrm{y}$ $1200^{\circ} \mathrm{C}$, con una velocidad de calentamiento de $2^{\circ} \mathrm{C} / \mathrm{min}$. En cada caso se evaluó el aspecto final del espécimen, el cambio de coloración, y las modificaciones en el peso y resistencia mecánica; además se evalúo la microestructura por medio de microscopia electrónica de barrido (SEM). El ensayo de resistencia a la compresión se realizó 24 horas después de retirado el espécimen del horno, utilizando una máquina de ensayos Universal INSTRON 3369 a una velocidad de desplazamiento de $1 \mathrm{~mm} / \mathrm{min}$; en todos los casos se utilizaron tres probetas. Para efectos comparativos se utilizaron pastas y morteros preparados a las 
mismas condiciones y expuestos a temperatura ambiente $\left(25^{\circ} \mathrm{C}\right)$.

\subsection{Técnicas experimentales utilizadas}

En el presente estudio se utilizaron las siguientes técnicas instrumentales.

- Difracción de Rayos X, la cual se llevó a cabo en un equipo PanAnalytical X'Pert MRD con radiación $\mathrm{Cu} \mathrm{K} \alpha$ a una velocidad de paso de 0,020 y en el rango $2 \theta=5-60^{\circ}$.

- Análisis térmico TG/DTG, se efectuó en un equipo simultáneo SDT-Q600 de TA Instruments. Las muestras se analizaron en atmósfera de nitrógeno, a una rata de calentamiento de $10^{\circ} \mathrm{C} / \mathrm{min}$ usando crisoles de alúmina.

- Microscopía electrónica de barrido (MEB) utilizando un equipo JEOL JSM-6490LV con un voltaje de aceleración de $5 \mathrm{kV}$.

\section{Resultados y discusión}

\subsection{Análisis por termogravimetría diferencial (DTG)}

Para conocer el comportamiento de estos materiales al estar expuestos a temperatura, inicialmente se realizó un ensayo de termogravimetría diferencial (DTG) para identificar los posibles cambios químicos e interpretar el desempeño del concreto cuando estuviera sometido a altas temperaturas (Rashad \& Zeedan, 2012; Janotka \& Mojumdar, 2005). En la figura 2 se presentan las curvas DTG, donde se pueden observar las siguientes transformaciones, un primer pico (1) atribuido a la pérdida del agua libre lo cual ocurre entre $50^{\circ} \mathrm{C}$ y $110^{\circ} \mathrm{C}$. El pico (2) ubicado a temperatura entre $115-120^{\circ} \mathrm{C}$ corresponde a la deshidratación parcial del silicato cálcico hidratado (CSH); es de suponer que el pico correspondiente a la descomposición de la Ettringita $\left(3 \mathrm{CaOAl}_{2} \mathrm{O}_{3} \cdot 3 \mathrm{CaSO}_{4} \cdot 32 \mathrm{H}_{2} \mathrm{O}\right)$ se encuentra solapado a estos dos primeros picos. El pico (3) entre $180-240{ }^{\circ} \mathrm{C}$ se atribuye a la deshidratación de los aluminatos cálcicos hidratados $(\mathrm{CAH})$ y el inicio de los silicoaluminatos cálcicos hidratados (CASH) y el pico (4), ubicado alrededor de $400-450{ }^{\circ} \mathrm{C}$ corresponde a la descomposición del hidróxido de calcio $(\mathrm{CH})$ a $\mathrm{CaO}$. Se observa en la figura 2 que las muestras con adición presentan menor cantidad de hidróxido de calcio $(\mathrm{CH})$ en comparación a las muestras sin adición, lo cual se puede atribuir al consumo del $\mathrm{CH}$ en la reacción con las adiciones, confirmando las características puzolánicas del FCC.

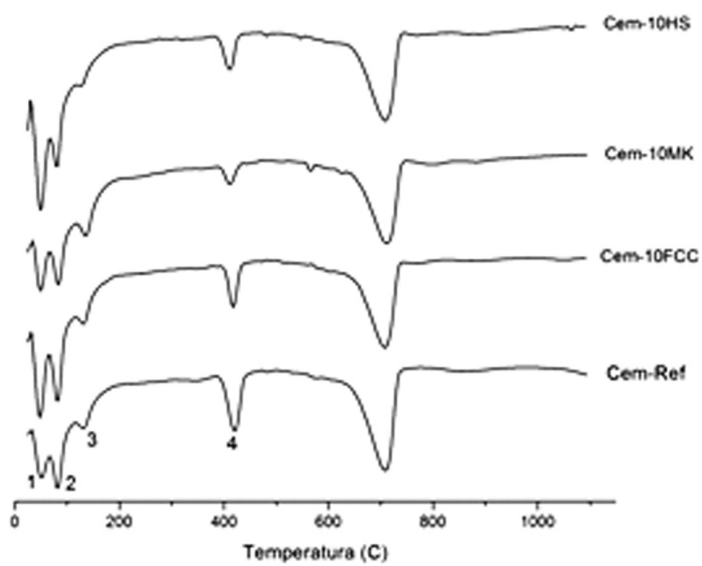

Figura 2. Curvas DTG para cem-ref, cem-10FCC, cem$10 M K$ y cem-10HS, después de 28 días de curado en agua con $\mathrm{Ca}(\mathrm{OH})_{2}$ y 15 dias al aire.

Finalmente, entre los 650 y $750^{\circ} \mathrm{C}$ aparece un pico en todas las mezclas, el cual es atribuible a la descarbonatación de la adición caliza presente en el cemento utilizado (tal como se puede constatar con el elevado porcentaje de pérdidas por ignición, ver tabla 1), sumado a la pérdida de agua de la descomposición del silicato cálcico hidratado. (Akca \& Zihnioğlu, 2013; Balazs \& Lubloy, 2012)

\subsection{Efecto de la temperatura en la resistencia a la compresión}

La Figura 3(a) presenta el comportamiento de la resistencia a la compresión de los diferentes morteros al ser expuestos a un rango de temperaturas entre $25^{\circ} \mathrm{C}-1200^{\circ} \mathrm{C}$. Aquí se expresa el índice de resistencia como la relación porcentual entre la resistencia obtenida a la temperatura Tx con la obtenida a la temperatura ambiente (Tamb) para cada mezcla. Se observa que los morteros expuestos a $100^{\circ} \mathrm{C}$ presentaron 

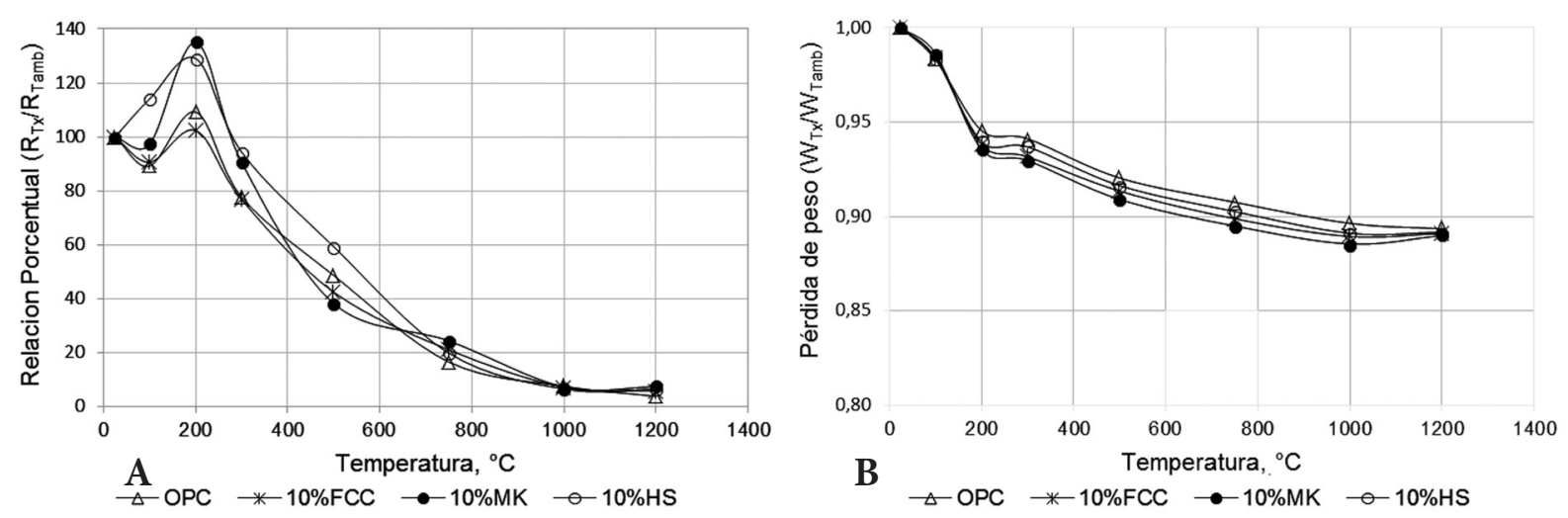

Figura 3. (a) Resistencia a la compresión y (b) Pérdida de peso de morteros referencia (OPC) y adicionados (10\%FCC, $10 \%$ MK y 10\%HS), después de ser expuestos a altas temperaturas.

una leve disminución en su resistencia mecánica con excepción de aquellos adicionados con el $10 \%$ de humo de sílice (HS), los cuales reportan una ganancia del $14,08 \%$. A $200^{\circ} \mathrm{C}$, se observa un incremento en esta propiedad para todas las mezclas analizadas, entre un 2,39 y $35,05 \%$, destacándose las adicionadas con MK y HS, concordando con lo reportado por Morsy (2008) y Poon \& Azhar (2003). Se atribuye este fenómeno a que el calor cataliza la hidratación de las partículas del cemento anhidro presente e incrementa las reacciones puzolánicas de las adiciones con el $\mathrm{CH}$ para dar lugar a la formación de $\mathrm{CSH}$ adicional, al generarse un efecto interno en el material tipo autoclave (Poon \& Azhar, 2003; Morsy, 2008). Otros autores sugieren que los grupos silanoles al perder parte del agua dan lugar a especies más fuertes tipo siloxano ( $\mathrm{Si}$ $\mathrm{O}-\mathrm{Si}$ ) con energías superficiales mayores que contribuyen al aumento de la resistencia (Uysal et al., 2012).

A partir de $200^{\circ} \mathrm{C}$ se observa una disminución continua en la resistencia a la compresión al incrementar la temperatura hasta los $1200^{\circ} \mathrm{C}$. A los $500^{\circ} \mathrm{C}$ se observa que las adiciones con $10 \%$ de humo de sílice presentan la menor perdida de resistencia $40,76 \%$ mientras que los morteros adicionados con $10 \% \mathrm{MK}, 10 \% \mathrm{FCC}$ y el cemento de referencia (Cem.Ref), presentan una pérdida de $61,79 \%, 57,59 \%$ y $51,38 \%$ respectivamente. Mehta \& Monterio (1997) plantean que la exposición de elementos cementicios a altas temperaturas tiene un efecto significativo en el engrosamiento de la estructura de poros, por lo cual se atribuye la pérdida resistente a este en lugar de a la descomposición del CSH a dicha temperatura. La descomposición del $\mathrm{CH}$, que se genera a temperaturas entre $430^{\circ} \mathrm{C}$ y $600^{\circ} \mathrm{C}$, tiene un efecto adicional en la pérdida de resistencia por debajo de $\operatorname{los} 600^{\circ} \mathrm{C}$. A partir de la temperatura de $750^{\circ} \mathrm{C}$ se observa que la menor resistencia $(8,7$ $\mathrm{MPa}$ ) la presentan los morteros Cem.Ref (OPC); a esta temperatura en particular la resistencia disminuye $83,47 \%$ mientras que los morteros adicionados con $10 \% \mathrm{FCC}, 10 \% \mathrm{MK}$ y $10 \% \mathrm{HS}$ presentan pérdidas de 78,98\%, 75,65\% y 80,20\% respectivamente. Este comportamiento inferior de los morteros de referencia puede atribuirse a la mayor disposición de $\mathrm{CH}$ con la que cuentan estas mezclas en comparación a los morteros con adición, lo cual se pudo apreciar en el estudio realizado por TG (Figura 2). Es de suponer que el mayor contenido de $\mathrm{CH}$ da lugar a una pérdida de agua enlazada superior y en consecuencia un mayor engrosamiento de la estructura siendo más evidente la perdida de resistencia y a su vez el incremento de la permeabilidad (Janotka \& Mojumdar, 2005) Cabe anotar, que a temperatura del orden de $750^{\circ} \mathrm{C}$, además de la descomposición del CSH, también se produce la descarbonatación de la caliza presente como adición inicialmente en el cemento utilizado. Pérdidas resistentes a temperaturas de hasta $800^{\circ} \mathrm{C}$ en orden similar han sido reportadas por otros investigadores para concretos con adiciones de escoria, cenizas volantes y otros tipos de adiciones (Uysal et al., 2012; Rashad \& Zeedan, 2012; Khan, 2013). 
En el rango de temperatura de $1000^{\circ} \mathrm{C}$ a $1200^{\circ} \mathrm{C}$ no se observan disminuciones drásticas en la resistencia a la compresión, revelando finalmente resistencias residuales comparadas a las obtenidas a temperatura ambiente de 7,58\%, 7,16\%, 7,24\% y $6,53 \%$ para los morteros OPC, $10 \%$ FCC, $10 \%$ MK y $10 \% \mathrm{HS}$ respectivamente. La pérdida de resistencia a estas temperaturas es debida también a la descomposición en alto grado del gel CSH (Heikal et al., 2013; Georgali \&Taskiridis, 2005; Poon et al., 2001)

\subsection{Pérdidas de peso y efectos físicos}

La modificación en la estructura de la matriz como consecuencia de la deshidratación en las fases del cemento durante la exposición a diferentes temperaturas se manifiesta en pérdidas de peso, cambio de color y presencia de grietas asociados a los cambios reportados en las propiedades mecánicas. En la Figura 3(b) se presentan las modificaciones en el peso de cada mezcla al ser expuestas a temperatura entre $25^{\circ} \mathrm{C}-1200{ }^{\circ} \mathrm{C}$. La pérdida de agua al exponer los morteros a la temperatura de $100{ }^{\circ} \mathrm{C}$, fue igual para todas las mezclas analizadas $(2 \%)$, esta puede considerarse debida a la pérdida de agua libre presente en los poros capilares. Se observa una disminución drástica del peso de las diferentes mezclas hasta $\operatorname{los} 200^{\circ} \mathrm{C}, 6 \%$ para los morteros Cem.Ref, Cem$10 \mathrm{FCC}$ y $\mathrm{Cem}-10 \mathrm{HS}$, y $7 \%$ para morteros con adición del 10\% MK. Este comportamiento puede estar asociado a la evaporación del agua químicamente enlazada. En el rango de $300^{\circ} \mathrm{C}$ a $1000{ }^{\circ} \mathrm{C}$ se observa una disminución en el peso, hasta alcanzar prácticamente un equilibrio entre $1000{ }^{\circ} \mathrm{C}$ y $1200{ }^{\circ} \mathrm{C}$. La pérdida de peso a 1000 ${ }^{\circ} \mathrm{C}$, puede atribuirse a la descomposición del gel silicato cálcico hidratado (CSH) (Heikal et al., 2013; Georgali \&Taskiridis, 2005; Poon et al., 2001).

Peng et al. (2001) realizaron estudios en pastas de cemento Portland donde comprobaron que el CSH inicia su proceso de descomposición a temperaturas superiores a los $600^{\circ} \mathrm{C}$, sin embargo es aún un tema controvertido ya que existen autores como Nijland \& Larbi, (2001), quienes proponen que esta descomposición puede dar inicio desde la temperatura de $180^{\circ} \mathrm{C}$, aunque sus efectos mayoritarios se produzcan a temperaturas superiores a $300^{\circ} \mathrm{C}$. Las pérdidas de peso a la temperatura de $750{ }^{\circ} \mathrm{C}$ para los materiales estudiados están en el rango de 10 a $11 \%$ y a 1000 ${ }^{\circ} \mathrm{C}$ no superan el $13 \%$.

La Figura 4 presenta las modificaciones de color asociadas a las temperaturas de ensayo. Los morteros expuestos a $200{ }^{\circ} \mathrm{C}$ no presentan ningún cambio en su coloración, lo cual indica la estabilidad de estos a esta temperatura. Los cambios de coloración comienzan a ser evidentes a partir de los $750{ }^{\circ} \mathrm{C}$. A esta temperatura las probetas Cem.Ref (OPC) revelan las primeras grietas, mientras que las muestras con adición no las presentaron demostrando una mayor estabilidad y su resistencia al fuego a esta temperatura, aunque el rango de pérdida de resistencia mecánica sea similar (75 a 84\%).

Algunos investigadores han reportado que las grietas altamente alargadas y la delaminación, en términos generales en los concretos se presentan a temperaturas superiores a los $800{ }^{\circ} \mathrm{C}$ (Bastami et al., 2011). Sin embargo en las probetas adicionadas y expuestas a $1000{ }^{\circ} \mathrm{C}$, a pesar de presentar una coloración más clara y algo más amarilla, no presentan fisuras. El agrietamiento de las probetas con adición de $10 \%$ FCC, $10 \% \mathrm{MK}$ y $10 \% \mathrm{HS}$ se hace visible a temperatura de $1200{ }^{\circ} \mathrm{C}$, entre estos los morteros con adición de MK presentaron la mayor fisuración. En general, puede afirmarse que las probetas presentan una buena integridad a estas altas temperaturas, aunque la reducción de su resistencia sea notable.

\subsection{Microestructura}

Las micrografías de las pastas referencia y adicionadas expuestas a $750{ }^{\circ} \mathrm{C}$ y $1200{ }^{\circ} \mathrm{C}$ se presentan en la figura 5. Las microestructuras de las diferentes mezclas expuestas a $750{ }^{\circ} \mathrm{C}$ revelan la presencia aun de los productos de hidratación tales como silicatos cálcicos hidratados $\mathrm{y}$ Etringita, sin embargo no se observan cristales de Portlandita $(\mathrm{CH})$, debido a que a esta temperatura ha tenido lugar la transformación de hidróxido de calcio $(\mathrm{CH})$ en $\mathrm{CaO}$, tal como se aprecia en la 

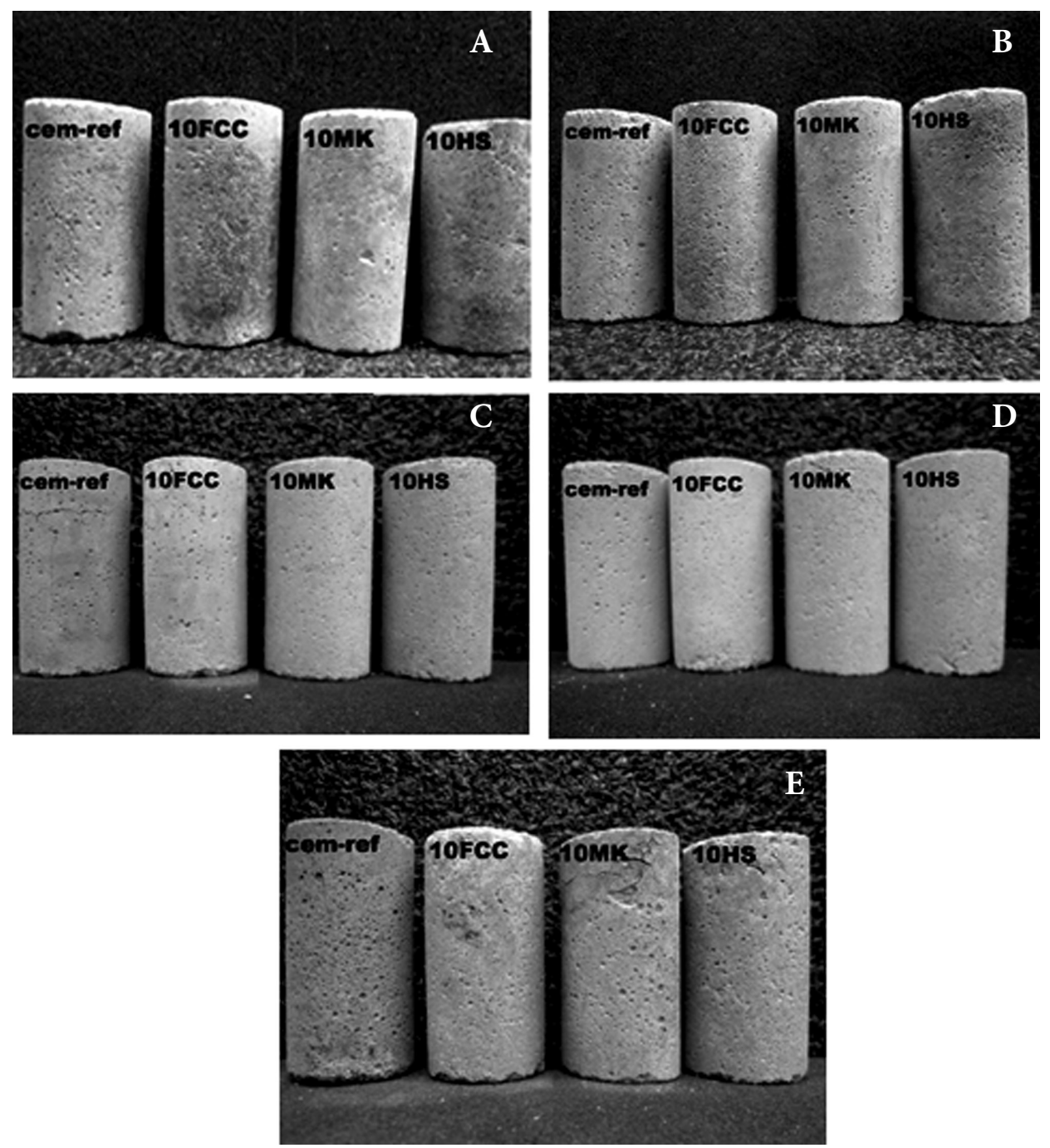

Figura 4. Cambios de apariencia y color con la temperatura en morteros referencia (100\%OPC), $10 \% \mathrm{FCC}, 10 \% \mathrm{MK}$ y $10 \% \mathrm{HS}$. a) $25^{\circ} \mathrm{C}$, b) $200^{\circ} \mathrm{C}$, c) $750^{\circ} \mathrm{C}$, d) $1000^{\circ} \mathrm{C}$ y e) $1200^{\circ} \mathrm{C}$.

Figura 2 (Rashad \& Zeedan, 2012). Se observa una matriz más homogénea en las probetas con adición y un mayor deterioro en la microestructura de las pastas de referencia (Cem.Ref)), lo cual puede explicar el menor comportamiento frente a la resistencia a la compresión al ser expuestas a esta temperatura (Figura 3a). A la temperatura de $1200{ }^{\circ} \mathrm{C}$ las micrografías SEM revelan que las fases hidratadas incluyendo el CSH aparecen como estructuras amorfas. También se evidencia la aparición de cristales redondeados los cuales pueden ser $\mathrm{C}_{2} \mathrm{~S}-\beta$, el cual es uno de los productos de descomposición del CSH a elevadas temperaturas (Turker et al., 2001; Rashad \& Zeedan, 2012). A esta temperatura $\left(1200^{\circ} \mathrm{C}\right)$, los espacios y porosidades en la pasta son mayores y se alcanza a observar la presencia de grietas (Akca \& Zihnioğlu, 2013).

\section{Conclusiones}

Las mezclas evaluadas pierden aproximadamente entre un 6 a 24\% de su resistencia a compresión a temperatura inferior a $300^{\circ} \mathrm{C}$, y entre un $40 \mathrm{y}$ $60 \%$ a temperaturas en el rango de 300 a $500{ }^{\circ} \mathrm{C}$. Luego de esta temperatura la caída resistente es significativa. Cabe resaltar que, a la temperatura de $750^{\circ} \mathrm{C}$ los morteros con adición presentan las menores pérdidas de resistencia a la compresión, confirmando una mayor estabilidad otorgada al 

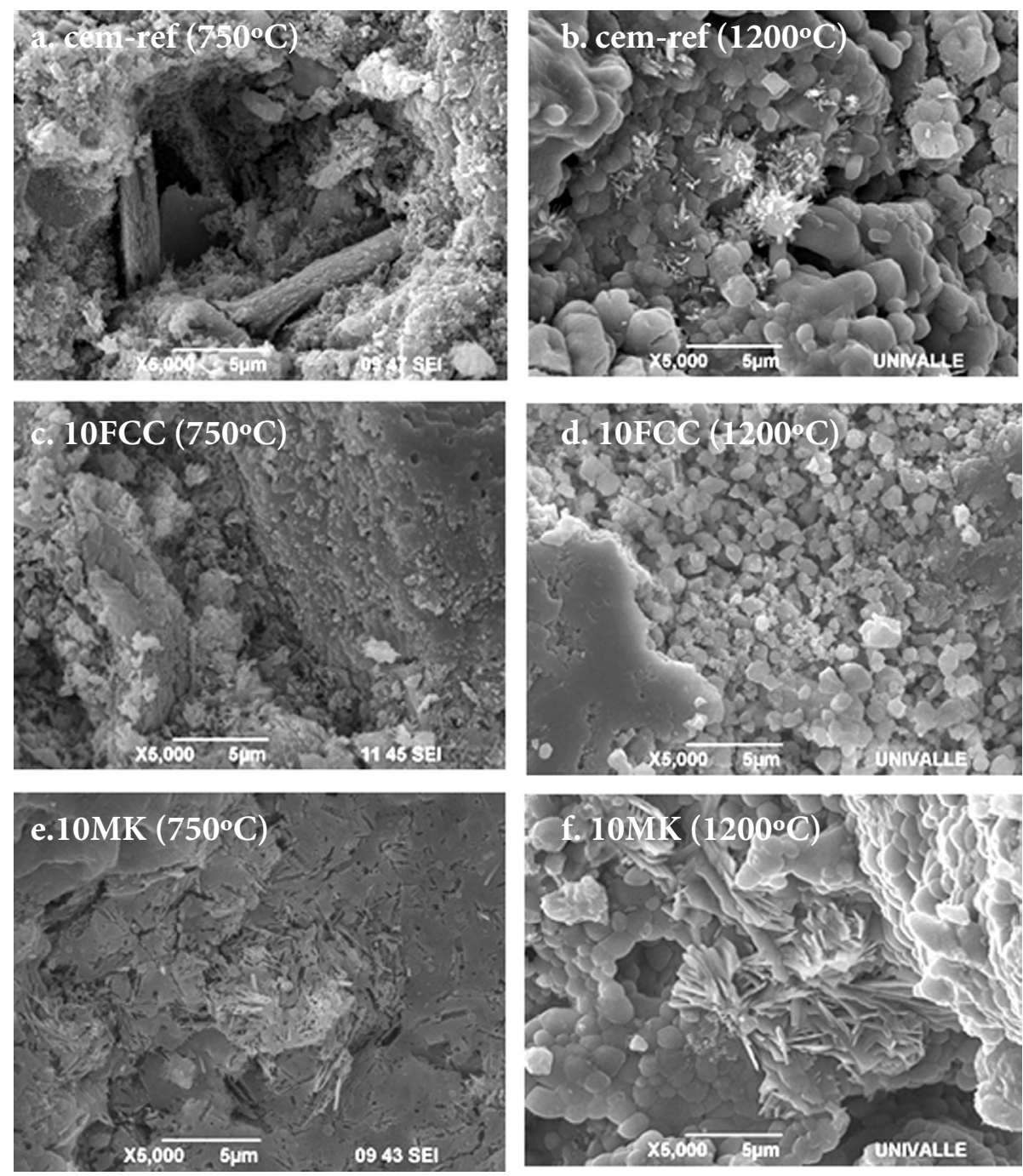

Figura 5. Micrografias SEM de pastas expuestas a temperaturas de $750^{\circ} \mathrm{C}$ y $1200^{\circ} \mathrm{C}$.

material al adicionar $10 \%$ de FCC, $10 \%$ de MK y $10 \%$ de HS. La resistencia residual del material adicionado con $10 \%$ FCC a $750^{\circ} \mathrm{C}$ es de 11,10 $\mathrm{MPa}$, un $26 \%$ superior a la reportada por el material de referencia (OPC). Los cambios de color con la temperatura resultan evidentes a los $750^{\circ} \mathrm{C}$, a esta misma temperatura se presentan grietas en los especímenes de OPC 100\%. Adicionalmente, se observa que la adición del 10\% de FCC retrasa la aparición de grietas en los elementos cementicios expuestos a altas temperaturas en comparación con aquellos sin adición. Cabe aclarar que no aparecen en ninguna de las probetas signos de delaminación.

En general, el mejoramiento de la resistencia a altas temperaturas del material adicionado conteniendo FCC, MK y HS, se atribuye a la densificación de la estructura de la pasta, tal como se observa en la micrografía SEM, y a la menor disponibilidad de $\mathrm{CH}$ en el sistema, lo cual se pudo apreciar por medio de la técnica de termogravimetría.

\section{Agradecimientos}

Los autores del presente artículo agradecen a la Universidad del Valle, Universidad Nacional de Colombia, al Centro de Excelencia en Nuevos Materiales (CENM) y al Departamento Administrativo de Ciencia, Tecnología e Innovación (Colciencias) por el apoyo en el desarrollo del presente estudio. 


\section{Referencias bibliográficas}

Akca, A. H., \& Zihnioğlu, N. O. (2013). High performance concrete under elevated temperatures. Construction and Building Materials 44, 317-328.

Ahmed, A.E, Al-Shaikh, A.H., \& Arafat, T.I. (1992). Residual compressive and bond strength of limestone aggregate concrete subjected to elevated temperatures. Magazine of Concrete Research 44 (159), 117-125.

Arioz, O. (2007). Effects of elevated temperatures on properties of concrete. Fire Safety Journal 42 (8), 516-522.

Aydin S. (2008). Development of a hightemperature-resistant mortar by using slag and pumice. Fire Safety Journal 43 (8), 610-617.

Aydin, S., Yazici, H., \& Baradan B. (2008). High temperature resistance of normal strength and autoclaved high strength mortars incorporated polypropylene and steel fibers. Construction and Building Materials 22 (4), 504-512.

Balazs, G.L., \& Lubloy, E. (2012). Reinforced concrete structures in and after fire. Concrete Structures, 72-80. http://fib.bme.hu/cs2012/ kicsom/cs2012web/lores/vb2012\%20angol\%20 BALAZS-BLOY\%20pp72-80.pdf

Bastami, M., Chaboki-Khiabani, A., Baghbadrani M., \& Kordi M. (2011). Performance of high strength concretes at elevated temperatures. Scientia Iranica 18 (5), 1028-1036.

Behnood, A., \& Ghandehari, M. (2008). Comparison of compressive and splitting tensile strength of high-strength concrete with and without polypropylene fibers heated to high temperatures. Fire Safety Journal 44 (8), 10151022.

Behnood, A., \& Ziari, H. (2008). Effects of silica fume addition and water to cement ratio on the properties of high-strength concrete after exposure to high temperatures. Cement \& Concrete Composites 30 (2), 106-112.
Chen, H.L., Tseng, Y.S, \& Hsu K.C. (2004). Spent FCC catalyst as a pozzolanic material for high-performance mortars. Cement and Concrete Composites 26 (6), 657-664.

De Souza, A. A. A., \& Moreno, A. L. (2010). The effect of high temperatures on concrete compression strength, tensile strength and deformation modulus. Revista IBRACON de Estruturas e Materiais 3 (4), 432-448.

Demirboga, R., Turkmen, I., \& Karakoc, M.B. (2007). Thermo-mechanical properties of concrete containing high volume mineral admixtures. Building and Environment 42 (1), 349-354.

Fernández Canovas, M. (1994). Patología y Terapéutica del Hormigón Armado. Madrid: Editorial Rugarte.

Georgali, B., \& Taskiridis, P.E. (2005). Microstructure of fire-damaged concrete, a case study. Cement and Concrete Composites 27 (2), 255-259.

Heikal, M., El-Didamony, H., Sokkary, T.M., \& Ahmed, I.A. (2013). Behavior of composite cement pastes containing microsilica and fly ash at elevated temperature. Construction and Building Materials 38, 1180-1190.

Heikal, M. (2000). Effect of temperature on the physico-mechanical and mineralogical properties of Homra pozzolanic cement pastes. Cement and Concrete Research 30 (11), 1835-1839.

Hossain K. M. A. (2006). High strength blended cement concrete incorporating volcanic ash: Performance at high temperatures. Cement and Concrete Composites 28 (6), 535-545.

Janotka, I., \& Mojumdar, S.C. (2005). Thermal analysis at the evaluation of concrete damage by high temperatures. Journal Thermal Analysis Calorimetry 81 (1), 197-203.

Kalifa, P., Chene, G. \& Galle C. (2001). Hightemperature behavior of HPC with polypropylene 
fibres: from spalling to microstructure. Cement and Concrete Research 31 (10), 1487-1499.

Khan, M. S., Prasad, J., \& Abbas, H. (2013). Effect of high temperature on high-volume fly ash concrete. Arabian Journal for Science and Engineering 38(6), 1369-1378.

Kodur, V. (2014). Properties of Concrete at elevated temperatures. Review Article. ISRN Civil Engineering Article ID468510, 15p. http:// dx.doi.org/10.1155/2014/468510

Mehta, P.K., \& Monterio, P.J.M. (1997). Concretemicrostructure, properties and materials. Chennai: Indian Concrete Institute.

Morsy, M.M., Shebl, S.S., \& Rashad, A.M. (2008). Effect of fire on microstructure and mechanical Properties of blended cement pastes containing Metakaolin and silica fume. Asian Journal of Civil Engineering (Building and Housing) 9 (2), 93-105.

Su, N., Fang, H-Y., Chen, Z-H., \& Liu, F-S. (2000). Reuse of waste cataysts from petrochemical industries for cement substitution. Cement and Concrete Research 30 (11), 1773-1783.

Naus, D. J. (2005). The Effect of Elevated Temperature on Concrete Materials and structures. A Literature Review. ORNL/TM-2005/553, Oak Ridge National Laboratory. http://info.ornl.gov/ sites/publications/files/Pub1043.pdf

Netinger, I., Kesegic, I., \& Guljas, I. (2011). The effect of high temperatures on the mechanical properties of concrete made with different types of aggregates. Fire Safety Journal 46 (7), 425-430.

Nijland, T., \& Larbi, J. (2001). Unraveling the temperature distribution in fire-damage concrete by means of PFm microscopy: Outline of the approach and review of potentially useful reactions. HERON journal 46 (4), 253-264.

Payá, J, Monzó, J, Borrachero, M.V., \& Velázquez S. (2003). Evaluation of the pozzolanic activity of fluid catalytic cracking catalyst residue (FC3R).
Thermogravimetric analysis studies on FC3RPortland cement pastes. Cement and Concrete Research 33 (4), 603-609.

Payá, J., Borrachero, M.V., Monzó, J., \& Soriano L. (2009). Estudio del comportamiento de diversos residuos de catalizadores de craqueo catalítico (FCC) en cemento Portland. Materiales de Construcción 59 (296), 37-52.

Peng, G.F., Chan, SYN., \& Anson, M. (2001). Chemical Kinetics of CSH Decomposition in hardened Cement Paste subjected to elevated Temperatures up to $800^{\circ} \mathrm{C}$. Advanced cement research $13,47-52$.

Pinto, C. A., Büchler, P. M., \& Dweck, J. (2007). Pozzolanic Properties of a Residual FCC Catalyst during the Early Stages of Cement Hydration Evaluation by thermal analysis. Journal of Thermal Analysis and Calorimetry 87 (3), 715720 .

Pliya, P, Beaucour, A-L., \& Noumowé A. (2011). Contribution of cocktail of polypropylene and steel fibres in improving the behaviour of high strength concrete subjected to high temperature. Construction and building materials 25 (4), 1926-34.

Poon, C.S., Azhar, S., Anson, M., \& Wong, Y.L. (2001) Comparison of the strength and durability performance of normal and high-strength pozzolanic concretes at elevated temperatures. Cement and concrete research 31 (9), 1291-1300.

Poon, S., \& Azhar, S. (2003). Deterioration and Recovery of Metakaolin Blended Concrete Subjected to High Temperature. Fire technology 39 (1), 35-45.

Rashad, A., \& Zeedan, S. (2012). A preliminary study of blended pastes of cement and quartz powder under the effect of elevated temperature. Construction and building materials 29, 672-681.

Sarshar, R., \& Khoury, G.A. (1993). Material and environmental factors influencing the compressive strength of unsealed cement paste 
and concrete at high temperatures. Magazine of Concrete Research 45, 51-61.

Su, N., Fang, H.Y., Chen, Z.H., \& Liu, F.S. (2000). Reuse of waste catalysts from petrochemical industries for cement substitution. Cement and Concrete Research 30, 1773-1783

Tanyildizi, H., \& Coskun, A., (2008). The effect of high temperature on compressive strength and splitting tensile strength of structural light weight concrete containing fly ash. Construction and building materials 22 (11), 2269-2275.

Torres, J., Baquero, E.A., \& Silva, A.R. (2009). Evaluación de la actividad puzolánica de un residuo de la industria del petróleo. Dyna 76 (158), 49-53.

Tseng, Y.S., Huang, C.L., \& Hsu, K.C. (2005). The pozzolanic activity of a calcined waste FCC catalyst and its effect on the compressive strength of cementitious materials. Cement and concrete research $35,782-787$.

Turker, P., Erdogdu, K., \& Erdogan B. (2001). Investigation of fire-exposed mortars with different types of aggregates. Cement concrete world 6, 52-67.
Uysal, M., Yilmaz, K., \& Ipek, M. (2012). Properties and behavior of self-compacting concrete produced with GBFS and FA additives subjected to high temperatures. Construction and Building Materials 28, 321-326.

Xiao, J., Falkner, H., (2006). On residual strength of high-performance concrete with and without polypropylene fibers at elevated temperatures. Fire safety journal 41 (2), 115-121.

Xu, Y., Wong, Y.L., Poon, C.S., \& Anson, M. (2001). Impact of high temperature on PFA concrete. Cement and concrete research 31, 1065-1073.

Yuksel, I., Siddique, R., \& Ozkan, O. (2011). Influence of high temperature on the properties of concretes made with industrial by-products as fine aggregate replacement. Construction and building materials 25, 967-72.

Yun-Sheng, T., Chen-Lin H., \& Kung-Chung, H. (2005). The pozzolanic activity of a calcined waste FCC catalyst and its effect on the compressive strength of cementitious materials. Cement and concrete research $35,782-787$. 